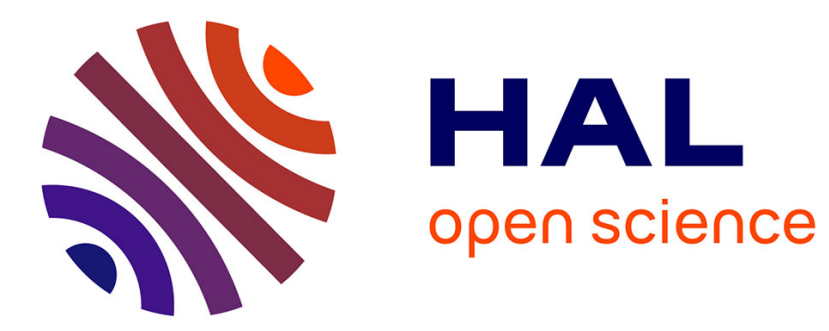

\title{
Etude des propriétés électriques des structures métal-polysiloxane-nGaAs
}

Y. Segui, B. Moret, D. Montalan

\section{To cite this version:}

Y. Segui, B. Moret, D. Montalan. Etude des propriétés électriques des structures métal-polysiloxane-nGaAs. Revue de Physique Appliquée, 1984, 19 (4), pp.325-331. 10.1051/rphysap:01984001904032500 . jpa-00245200

\section{HAL Id: jpa-00245200 https://hal.science/jpa-00245200}

Submitted on 1 Jan 1984

HAL is a multi-disciplinary open access archive for the deposit and dissemination of scientific research documents, whether they are published or not. The documents may come from teaching and research institutions in France or abroad, or from public or private research centers.
L'archive ouverte pluridisciplinaire HAL, est destinée au dépôt et à la diffusion de documents scientifiques de niveau recherche, publiés ou non, émanant des établissements d'enseignement et de recherche français ou étrangers, des laboratoires publics ou privés. 
Classification

Physics Abstracts

$73.40 \mathrm{Q}-73.60 \mathrm{H}-52.80 \mathrm{H}$

\title{
Etude des propriétés électriques des structures métal-polysiloxane-nGaAs
}

\author{
Y. Segui, B. Moret et D. Montalan \\ Laboratoire de Génie Electrique, 118, route de Narbonne, 31062 Toulouse Cedex, France
}

(Reçu le 25 juillet 1983, révisé le 26 décembre, accepté le 5 janvier 1984)

\begin{abstract}
Résumé. - On montre que les films de polysiloxane déposés à température ambiante par plasma réactif sur des substrats de GaAs sont une alternative possible pour la passivation des composants à base de GaAs. L'étude des phénomènes de transport montre qu'il s'agit d'un excellent isolant jusqu'à des champs de $5 \times 10^{6} \mathrm{~V} / \mathrm{cm}$. Les propriétés de l'interface polysiloxane-GaAs sont caractérisées par une distribution en $U$ des états de surface avec un minimum situé à $10^{12} \mathrm{~cm}^{-2} \mathrm{eV}^{-1}$. Les structures étudiées ont une bonne stabilité sous humidité relative de $80 \%$ et un champ continu appliqué de $1,3 \times 10^{6} \mathrm{~V} / \mathrm{cm}$.
\end{abstract}

\begin{abstract}
It is shown that plasma deposited polysiloxane films on GaAs substrate at room temperature can be considered as a possible candidate for the passivation of $\mathrm{GaAs}$ devices. The study of transport phenomena shows that the polysiloxane films exhibit very good insulating properties up to electric field as high as $5 \times 10^{6} \mathrm{~V} / \mathrm{cm}$. The electrical properties of the interface polysiloxane-GaAs are characterized by a $U$ shaped distribution of interface states in the gap. The minimum value of the distribution is found to be $10^{12} \mathrm{~cm}^{-2} \mathrm{eV}^{-1}$. These structures are stable under the effects of $80 \%$ of relative humidity and $1.3 \times 10^{6} \mathrm{~V} / \mathrm{cm}$ applied to the gate.
\end{abstract}

\section{Introduction.}

Les films minces d'oxyde ou de nitrure sont largement utilisés pour les fonctions d'isolation ou de passivation dans les dispositifs à semiconducteur.

Dans le cas de l'arséniure de gallium, le problème se pose de manière plus complexe car d'une part sa température d'évaporation nécessite l'emploi d'une méthode de dépôt à basse température et d'autre part il semble que la physique de la surface de GaAs conduise à un pic de densité d'états qui ancre le niveau de Fermi dans la moitié inférieure de la bande interdite quelle que soit la nature du matériau déposé en surface [1]. Ces considérations nous ont conduit à élaborer et tester une interface GaAs/Isolant dans laquelle ce dernier est un polymère organométallique déposé par plasma froid basse fréquence [2].

\section{Dépôt plasma d'un film organométallique.}

Le dépôt du film polymère est réalisé par création d'un plasma basse fréquence dans des vapeurs du monomère basse pression. Le réacteur utilisé est de type coplanaire. Les paramètres les plus importants qui régissent la croissance du film solide sur un substrat à partir du plasma sont : la pression partielle et le débit de monomère dans le réacteur, le courant et la fréquence de la décharge, la température du substrat ainsi que les facteurs géométriques [3, 4]. Le monomère choisi pour cette étude est l'hexaméthyldisiloxane $\left(\mathrm{CH}_{3}\right)_{3} \mathrm{Si}-\mathrm{O}-\mathrm{Si}-\left(\mathrm{CH}_{3}\right)_{3}$. Les raisons de ce choix sont la stabilité thermique élevée du polymère obtenu (stable jusqu'à plus de $400^{\circ} \mathrm{C}$ ), et la pression de vapeur du monomère qui autorise des dépôts à température ambiante à des pressions de 0,2 à $0,5 \mathrm{~T}$ avec des vitesses de croissance convenables. Le mécanisme de la polymérisation par plasma sur un substrat métallisé a fait l'objet d'une étude détaillée [5]. La croissance sur un substrat de GaAs obéit qualitativement aux mêmes lois. La figure 1 montre que dans la gamme d'épaisseur utilisée la croissance est linéaire avec le temps, ce qui permet de définir des taux de croissance. La figure 2 montre l'effet de la pression sur la vitesse de croissance. Cette courbe en cloche est bien modélisée par l'approche du mécanisme de dépôt décrit en [1]. L'épaisseur du film déposé est uniforme à quelques $\%$ près et dans la gamme d'épaisseur $1000 \AA-1 \mu \mathrm{m}$ aucun problème n'a été rencontré, même lorsque l'échantillon est soumis à des chocs thermiques répétés entre $77 \mathrm{~K}$ et $473 \mathrm{~K}$. 


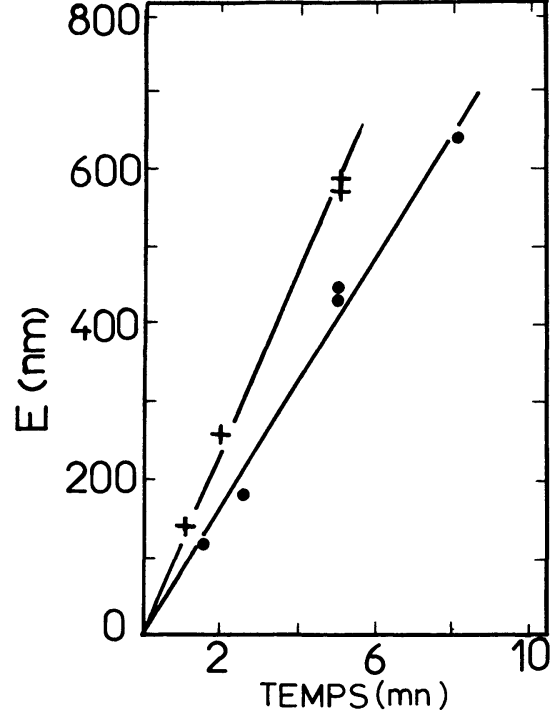

Fig. 1. - Variation de l'épaisseur de polysiloxane déposée en fonction du temps : courant de la décharge $0,17 \mathrm{~mA} / \mathrm{cm}^{2}$, tension de la décharge $0,17 \mathrm{~mA} / \mathrm{cm}^{2}$, tension de la décharge $340 \mathrm{~V}_{\text {eff }}$, pression partielle du monomère : $0,3 \mathrm{~T},+0,5 \mathrm{~T}$.

[Thickness of the plasma deposited polysiloxane as a function of time : discharge current $0.17 \mathrm{~mA} / \mathrm{cm}^{2}$, discharge voltage $340 \mathrm{~V}$ r.m.s., partial pressure of the monomer : $0.3 \mathrm{~T}$, $+0.5 \mathrm{~T}$.]

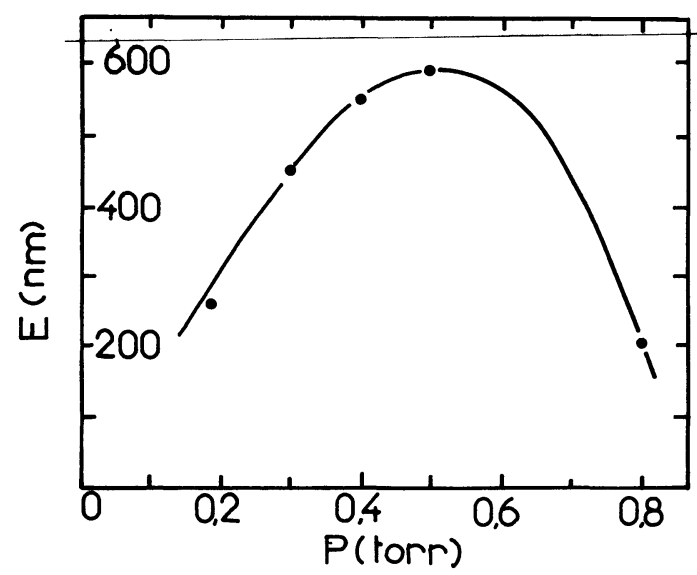

Fig. 2. - Influence de la pression partielle de monomère sur l'épaisseur de polymère déposé. Temps de dépôt : $5 \mathrm{~min}$.

[Effect of the partial pressure of the monomer on the thickness of the polymer. Deposition time $5 \mathrm{~min}$.]

\section{Propriétés électriques intrinsèques des films de polysiloxane.}

Lorsqu'on applique un échelon de tension à un diélectrique placé entre deux électrodes et que l'on mesure le courant résultant de cette contrainte on observe en général un courant variable dans le temps suivi d'un régime permanent [6].
En ce qui concerne les transitoires, trois types de comportement ont été mis en évidence.

Pour les champs faibles $E<2 \times 10^{5} \mathrm{~V} / \mathrm{cm}$, les courbes $\log I-\log t$ (Fig. 3) laissent apparaître deux régimes linéaires de pente différente. D'autre part les courants d'absorption et de résorption sont égaux en valeur absolue. Ce phénomène est caractéristique d'un comportement de type dipolaire [7].

Pour des champs plus élevés, les courants restent linéaires dans un diagramme $\log I-\log t$ (Fig. 4) mais ne sont plus superposables. Enfin pour des champs supérieurs à $10^{6} \mathrm{~V} / \mathrm{cm}$ le courant en fonction du temps présente l'allure indiquée sur la figure 5 .

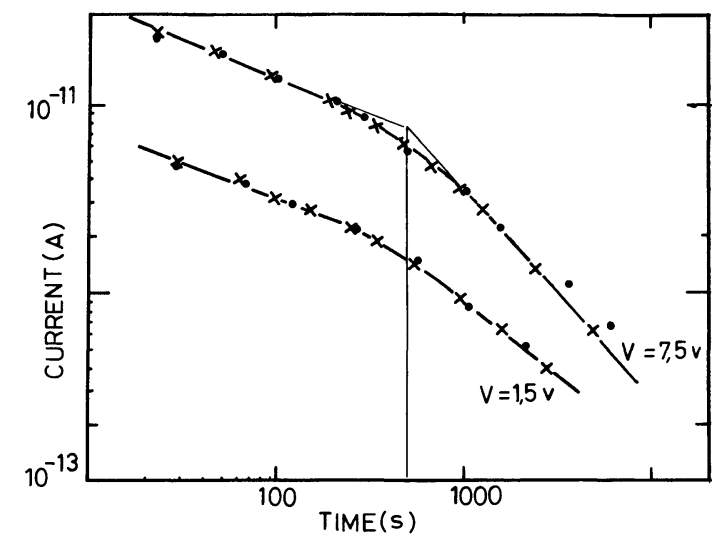

Fig. 3. - Réponse du diélectrique à un échelon de tension dans un diagramme Log-Log. Les courants d'absorption $(\mathrm{X})$ et de résorption (๑) sont superposables. Epaisseur du diélectrique : $5800 \AA$

[Log-Log plot of the dielectric response to a step voltage. Absorption (X) and resorption ( $(0)$ currents are superposable. Dielectric thickness : $5800 \AA$.]

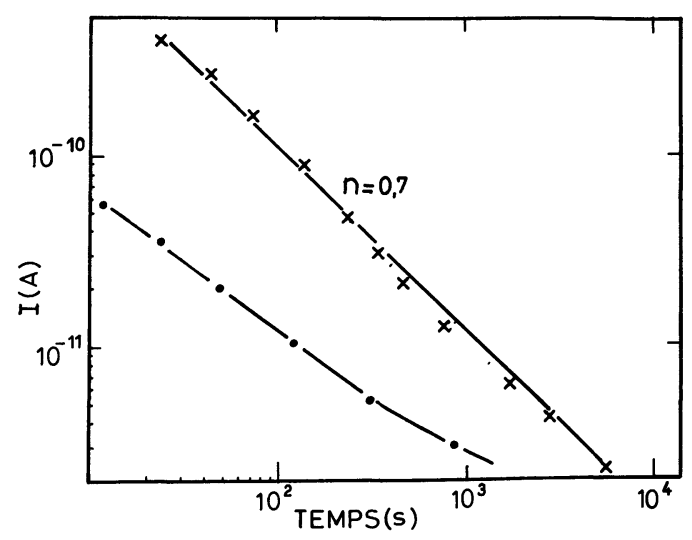

Fig. 4. - Courants d'absorption (X) et de décharge (O) en fonction du temps lorsqu'un champ de $3 \times 10^{5} \mathrm{~V} / \mathrm{cm}$ est appliqué à l'échantillon.

[Absorption (X) and discharging (๑) currents when a field of $3 \times 10^{5} \mathrm{~V} / \mathrm{cm}$ is applied to the sample.] 


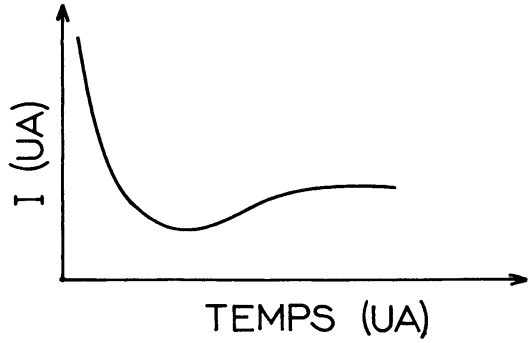

Fig. 5. - Allure de courbes $I(t)$ lorsqu'un échelon de $10^{6} \mathrm{~V} / \mathrm{cm}$ est appliqué au diélectrique.

[Typical $I(t)$ response when a field of $10^{6} \mathrm{~V} / \mathrm{cm}$ is applied to the dielectric.]

Ces deux derniers faits expérimentaux sont interprétés comme étant dus à une polarisation ionique qui peut modifier les conditions électriques à l'interface électrode-isolant et induire une injection électronique. Le courant représenté en 5 serait donc la résultante d'un courant ionique décroissant dans le temps et d'un courant électronique croissant.

Les courants permanents sont représentés sur la figure 6 en fonction de la racine carrée de la tension appliquée pour un échantillon de $1 \mu \mathrm{m}$ d'épaisseur. A température ambiante pour un champ de $10^{6} \mathrm{~V} / \mathrm{cm}$, le courant de fuite est de $8,8 \times 10^{-11} \mathrm{~A} / \mathrm{cm}^{2}$ ce qui conduit à une résistivité de $5 \times 10^{15} \Omega . \mathrm{cm}$.

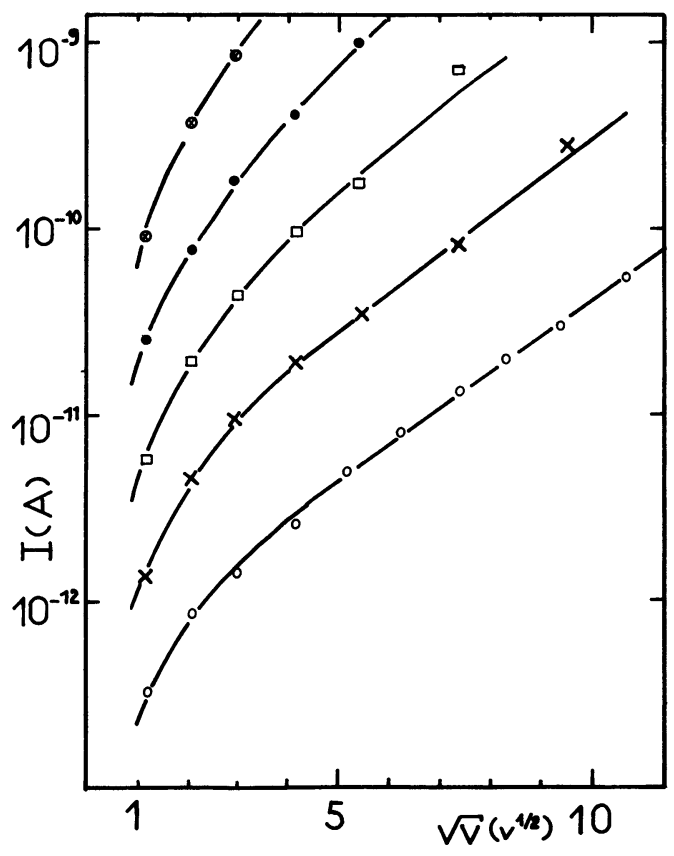

Fig. 6. - Courants permanents (ou de fuites) du polysiloxane en fonction de la tension appliquée. Epaisseur du diélectrique : $10000 \AA$; Températures : (O) $23{ }^{\circ} \mathrm{C},(\times) 41^{\circ} \mathrm{C}$, (口) $62^{\circ} \mathrm{C},(\bullet) 83^{\circ} \mathrm{C},(\otimes) 107^{\circ} \mathrm{C}$.

[Steady state (or leakage) currents of the polysiloxane films as a function of the applied voltage. Dielectric thickness : $10000 \AA$; Temperatures : (O) $23^{\circ} \mathrm{C},(\times) 41^{\circ} \mathrm{C},(\square) 62^{\circ} \mathrm{C}$, (•) $\left.83^{\circ} \mathrm{C},(\otimes) 107^{\circ} \mathrm{C}.\right]$
Le champ de rupture diélectrique est de l'ordre de $5 \times 10^{6} \mathrm{~V} / \mathrm{cm}$. Il est à noter que jusqu'à la rupture, le diélectrique reste un bon isolant et ne présente pas de croissance quasi exponentielle du courant de fuite.

$\mathrm{Au}$ niveau des propriétés électriques intrinsèques du polysiloxane déposé par plasma, on peut conclure qu'il se comporte comme un excellent isolant. Le seul point inquiétant dans l'optique de la passivation est la migration ionique que nous avons mise en évidence pour des champs supérieurs ou égaux à $3 \times 10^{5} \mathrm{~V} / \mathrm{cm}$.

\section{Etude des propriétés électriques de l'interface poly- siloxane-GaAs.}

4.1 Mesure De LA COURBE DE CAPACITÉ DYNAMIQUe. - L'outil d'investigation utilisé est la caractéristique $C(V)$ des structures Métal-Polysiloxane-nGaAs(MPS).

Nous disposons de deux types de système de mesure :

- pour une fréquence fixe de $1 \mathrm{MHz}$, nous utilisons un capacimètre BOONTON type $72 \mathrm{BD}$;

- pour une gamme de fréquence allant de $1 \mathrm{kHz}$ à $100 \mathrm{kHz}$, la mesure de la capacité est assurée par un détecteur de phase PAR type 129 A.

Les caractéristiques $C(V)$ des capacités MPS se présentent sous forme d'un cycle d'hystérésis. Leur sens initial est celui des aiguilles d'une montre, mais après plusieurs balayages en tension, le sens de parcours s'inverse. On remarque également que la largeur du cycle est sensible à la vitesse de balayage (Fig. 7).

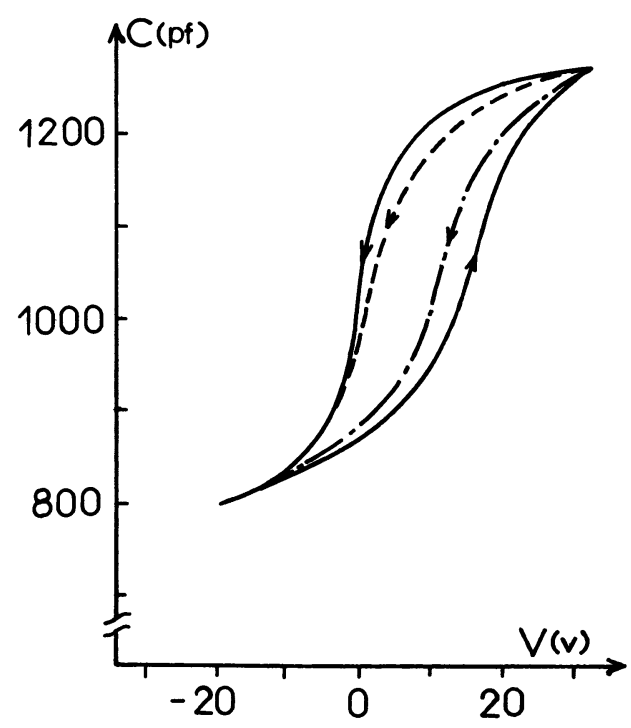

Fig. 7. - Caractéristiques $C(V)$ à $1 \mathrm{MHz}$ d'une structure Al-Polysiloxane-nGaAs à différentes vitesses de balayage : $-0,9 \mathrm{~V} / \mathrm{s}, \cdots .-4,5 \mathrm{~V} / \mathrm{s}, \ldots .-25 \mathrm{~V} / \mathrm{s}$.

$[C(V)$ curves at $1 \mathrm{MHz}$ of an Al-Polysiloxane-nGaAs structure at various sweep rates : $0.9 \mathrm{~V} / \mathrm{s}, \ldots .$. $4.5 \mathrm{~V} / \mathrm{s},-$. $-25 \mathrm{~V} / \mathrm{s}$.] 
La première remarque indique que le premier phénomène perceptible est un mécanisme d'échange de charges avec le semiconducteur puis par suite de la dissymétrie de la tension de balayage par rapport au zéro de tension, on assiste à l'effet du drainage qui amène les charges internes du polymère dans la région de l'interface avec le GaAs.

L'élargissement du cycle lorsque la vitesse de balayage diminue est cohérente avec cette dernière hypothèse.

4.2 ETUDES DES CARACTÉRISTIQUES $C(V)$ EN FONCTION DE LA FRÉQUENCE. - Les résultats présentés sont obtenus pour des substrats de $\mathrm{nGaAs}(100)$ non intentionnellement dopés, $N_{\mathrm{D}}=1,8$ à $3 \times 10^{16} \mathrm{~cm}^{-3}$ afin que les variations de la valeur des capacités dynamiques soient significatives.

La figure 8 montre les résultats obtenus pour un domaine de fréquences se situant entre $70 \mathrm{kHz}$ et $1 \mathrm{MHz}$. On observe :

- une importante dispersion en fréquence côté accumulation et plus faible côté inversion,

- existence bien marquée de paliers de capacité aussi bien côté accumulation que côté inversion.

Ces résultats sont attribués à la présence d'une forte densité d'états de surface à l'interface isolant semiconducteurs. Notons par ailleurs que des résultats analogues ont été reportés dans la littérature pour d'autres systèmes isolant-GaAs $[8,9]$.

Nous avons évalué la distribution et la densité de ces états par ta méthode de Terman [10] appliquee aux courbes $C(V)$ obtenues par une fréquence de $1 \mathrm{MHz}$.

Cette estimation ne tient pas compte des variations de la valeur de la permittivité relative de l'isolant car elles sont négligeables dans le domaine de fréquence recouvert.

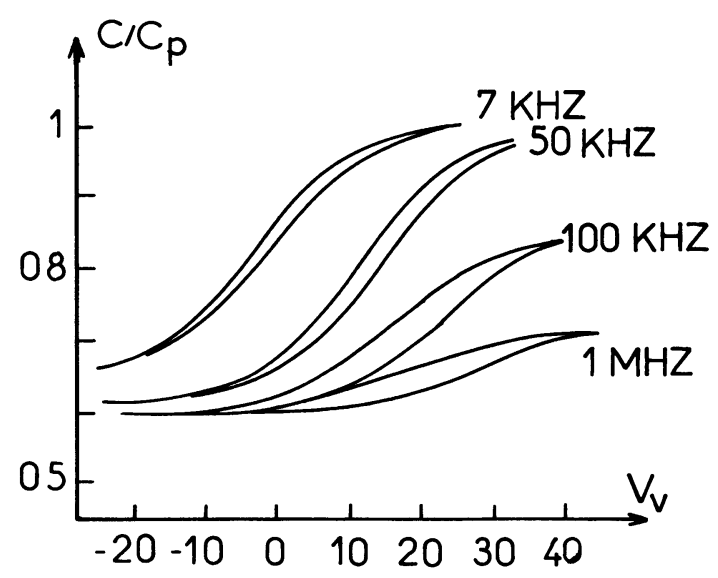

Fig. 8. - Courbes $C(V)$ à différentes fréquences de mesures d'une structure M-Polysiloxane-nGaAs $\left(N_{\mathrm{D}}=3 \times\right.$ $\left.10^{16} \mathrm{~cm}^{-3} ; d=900 \AA\right)$.]

[The $C(V)$ dependence at various measuring frequencies for an M-Polysiloxane-nGaAs capacitor $\left(N_{\mathrm{D}}=3 \times\right.$ $\left.10^{16} \mathrm{~cm}^{-3} ; d=900 \AA\right)$.]
Il convient de remarquer que la distribution des états de surface ne peut être déterminée que dans la partie inférieure de la bande interdite du semiconducteur. En effet, les valeurs de la capacité mesurées nous montrent que, pour une fréquence de $1 \mathrm{MHz}$, la valeur du potentiel de surface $\psi_{\mathrm{s}}$ varie entre $-0,6 \mathrm{eV}$ et $-1 \mathrm{eV}$ lors de l'application de la rampe de tension.

Les densités d'états de surface $N_{\mathrm{ss}}$ pour les systèmes M-P-nGaAs sont représentées sur la figure 9. Ces valeurs tiennent compte de la présence de charges dans le polymère.

Les valeurs de $N_{\mathrm{ss}}$ obtenues vers le milieu de la bande interdite, de l'ordre de $10^{13} \mathrm{~cm}^{-2} \mathrm{eV}^{-1}$ sont en bon accord avec les résultats trouvés dans la littérature [11-14].

Il existe un désaccord sur la localisation en énergie de cette distribution car la position des états dans la bande interdite est directement liée à la connaissance de $\psi_{\mathrm{s}}$ et dépend donc de la technique utilisée pour sa détermination.

L'imprécision induite sur la valeur du potentiel de surface par notre méthode de mesure n'excède pas $5 \%$.

4.3 MODÉLISATION DU COMPORTEMENT ÉLECTRIQUE DES STRUCTURES M-P-nGaAs. - Le modèle proposé pour rendre compte du comportement électrique de notre structure est schématisé sur la figure 10 , avec :

$R_{\mathrm{C}}, C_{\mathrm{P}}$ : résistance et compacité relatives au polymère. $R_{\mathrm{S}}, C_{\mathrm{S}}$ : résistance et compacité relatives aux états de surface.

$C_{\mathrm{D}} \quad$ : capacité du semiconducteur.

Les courbes représentatives de $C_{\mathrm{acc}} / C_{\mathrm{P}}=f(\omega)$ et de $C_{\text {inv. }} / C_{\mathrm{P}}=f(\omega)$, obtenues à partir de ce modèle sont en bon accord avec les points expérimentaux. Cependant, cet accord est obtenu en supposant une dispersion en fréquence de $\tau_{0}=R_{\mathrm{S}} C_{\mathrm{S}}$ (Fig. 11).

La constante de temps des états de surface $\tau_{\mathbf{S}}$ est évaluée par la méthode de la conductance en inversion.

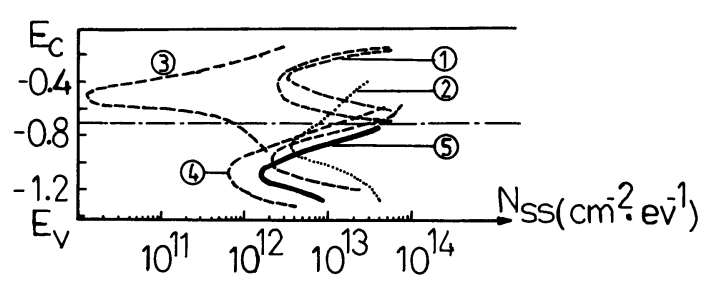

Fig. 9. - Distribution en énergie des états d'interfaces dans la bande interdite du GaAs. Comparaison entre le système polysiloxane-nGaAs et divers résultats rapportés dans la littérature [11]. Courbes $1 \rightarrow 4:$ [11], Courbe $5:$ polysiloxane.

[The interface state distribution in the energy gap. Comparaison between the polysiloxane-nGaAs structures and various results reported in the literature [11]. Curves $1 \rightarrow 4$ : [11], curve 5 : polysiloxane.] 


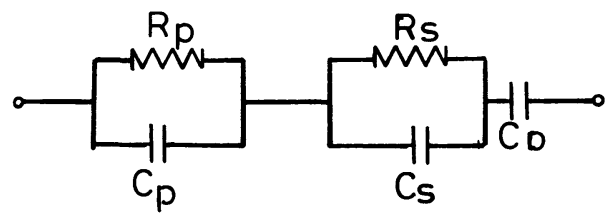

Fig. 10. - Schéma du circuit électrique utilisé pour modéliser la dispersion en fréquence des capacités MIS.

[Equivalent circuit modelling the frequency dispersion of MIS capacitors.]

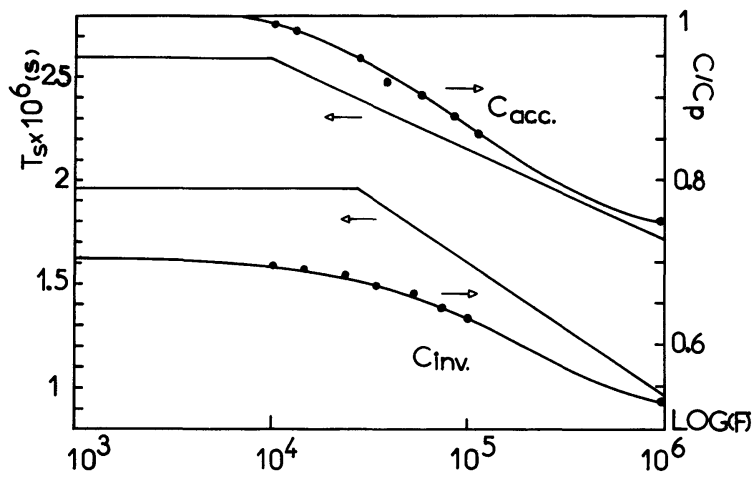

Fig. 11. - Variation de la capacité, normalisée par la valeur limite basse fréquence, en fonction de la fréquence dans le cas de " l'accumulation » et de "l'inversion »: —— théorie, - résultats expérimentaux.

[The capacitance, normalized by the low frequency limit, vs. frequency for accumulation and inversion : - theoretical curve; • experimental results.]

Pour cela, nous avons tracé la courbe représentant $G_{\mathrm{SS}}(\omega) / \omega=f(\omega)$ à une tension donnée. $G_{\mathrm{SS}}$ est la conductance des états de surface.

Le maximum de cette courbe correspond à une pulsation $\omega_{\mathrm{m}}$ avec $\omega_{\mathrm{m}} \times \tau_{\mathrm{s}}=1$ d'où la valeur de $\tau_{\mathrm{s}}$

Cette courbe a été tracée (Fig. 12) pour une tension égale à $-10 \mathrm{~V}$ qui met la structure en état d'inversion.

Pour des tensions correspondant au régime de déplétion, les courbes représentant $\left(G_{\mathrm{SS}} / \omega\right)=f(\omega)$ ne sont plus symétriques et ne permettent pas de déterminer $\tau_{\mathbf{s}}$; en effet cette méthode n'est acceptable que pour des densités d'états peu importantes.

La constante de temps des états de surface, $\tau_{\mathrm{s}}$ est trouvée égale à $2 \times 10^{-6} \mathrm{~s}$ mais la largeur du pic suggère l'existence d'une dispersion de $\tau_{\mathrm{s}}$ en fréquence. Parmi les mécanismes reponsables de cette dispersion nous pouvons retenir :

- l'échange par effet Tunnel entre le semiconducteur et des pièges situés dans l'isolant;

- les fluctuations du potentiel de surface dues, d'une part à une distribution en énergie de la densité d'états de surface et d'autre part à des charges distribuées dans l'isolant.

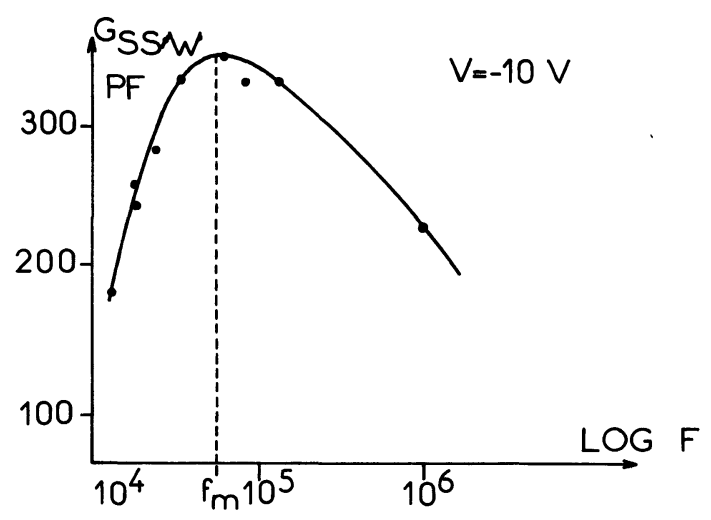

Fig. 12. - Variation de la conductance d'une structure M-polysiloxane-nGaAs en inversion en fonction de la fréquence.

[Frequency dependence of the conductance for an M-polysiloxane-nGaAs capacitor biased in inversion.]

La connaissance des valeurs de $\tau_{\mathrm{s}}$ et $\psi_{\mathrm{s}}$ nous permet d'avoir une estimation de la valeur de la section efficace de capture des trous. Nous trouvons des valeurs de l'ordre de quelque $10^{-15} \mathrm{~cm}^{2}$ qui sont celles habituellement rencontrées dans la littérature $[15,16]$.

\section{Influence de l'humidité sur les caractéristiques du polymère et de l'interface $\mathrm{GaAs}$.}

5.1 INFLUENCE DE L'HUMIDITÉ SUR LES PROPRIÉTÉS INTRINSÈQUES DU POLYSILOXANE. - Les films de polysiloxane sont déposés sur un support en verre, métallisés à l'or et placés dans une cellule d'essai dans laquelle le taux d'humidité relative peut être fixé aux valeurs de $20 \%, 48 \%$ et $80 \%$.

Une analyse par spectroscopie infrarouge montre à travers l'évolution du pic correspondant aux liaisons $\mathrm{OH}$ que le polysiloxane est sensible à l'humidité à partir d'un taux égal à $80 \%$. En ce qui concerne la permittivité, on a pu mettre en évidence qu'un recuit d' $1 \mathrm{~h} 30$ en atmosphère d'azote amenait le taux de variation de ce paramètre à une valeur de $2,6 \%$ pour une exposition d'une dizaine d'heures à $80 \%$ d'humidité relative.

5.2 INFLUENCE DE L'HUMIDITÉ SUR L'INTERFACE POLYSILOXANE-GaAs. - Nous n'avons observé aucune modification des caractéristiques $C(V)$ après exposition prolongée en atmosphère ayant des taux d'humidité de $20 \%$ et $48 \%$ pour des structures Au-P-GaAs et Au-P-Si.

Pour un taux d'humidité de $80 \%$ nous avons pu observer pour les deux types de structure une translation de la courbe $C(V)$ vers les tensions positives correspondant à une variation de charge négative ramenée à l'interface d'environ $2 \times 10^{11}$ charges $\mathrm{cm}^{-2}$

Les structures ont été exposées à une humidité importante $(80 \%)$ sous une contrainte de tension 
positive induisant un champ électrique de l'ordre de $10^{6} \mathrm{~V} / \mathrm{cm}$.

L'évolution dans le temps des courbes $C(V)$ est représentée sur la figure 13. On observe l'existence d'une dérive des courbes $C(V)$ vers des tensions négatives au début de la contrainte, puis le sens de la dérive s'inverse.

La dérive observée lors des premiers temps de l'application d'un champ positif révèle un accroissement de charges positives à l'interface où la migration des charges négatives conduisant à un abaissement du nombre de charges négatives présentes à l'interface. Il s'agit vraisemblablement de la migration des ions $\mathrm{OH}^{-}$vers l'électrode métallique, ce qui rend la charge restante localisée près du semiconducteur davantage positive et provoque la dérive des courbes $C(V)$ vers les tensions négatives dans un premier temps.

Nous remarquons ensuite une allure différente de la dérive des courbes $C(V)$, en effet le sens de la dérive s'inverse. Pour expliquer la remontée de la courbe plusieurs mécanismes sont envisageables :

- L'air humide est obtenu en faisant barboter l'air dans une solution saturée de chlorure de baryum. $\mathrm{BaCl}$. Après exposition à l'atmosphère humide des structures, des ions $\mathrm{OH}^{-}$sont présents dans le polymère mais on peut considérer aussi que des ions positifs $\mathrm{Ba}^{++}$ont pénétré dans le polysiloxane. Lors de l'application d'une tension positive, ces charges positives s'accumulent à l'interface polymèresemicon ucteur. Après la migration d'une partie des ions $\mathrm{OH}^{-}$, il peut y avoir une injection par le semiconducteur d'électrons qui neutralisent les charges positives, d'où la remontée de la courbe représentant la dérive des courbes $C(V)$, au régime des temps longs.

- La deuxième hypothèse est que le mécanisme d'injection par le semiconducteur a lieu dès les premiers temps de l'application de la polarisation positive, sans que la présence d'ions $\mathrm{Ba}^{++}$due à l'exposition à l'humidité soit envisagée. Il faut alors considérer que la constante de temps du mécanisme d'injection des électrons est plus faible que celle de la migration des ions $\mathrm{OH}^{-}$à travers le polymère. Cela paraît peu probable car les charges ioniques ont une mobilité faible.

- Le troisième mécanisme possible consiste en une injection d'électrons à partir du semiconducteur au régime des temps longs. Cette injection est rendue possible par la modification de la barrière à l'interface polymère-semiconducteur, due à la migration des ions $\mathrm{OH}^{-}$. Alors que les ions $\mathrm{OH}^{-}$peuvent migrer vers l'électrode métallique, les électrons sont piégés et restent près de l'interface.

En atmosphère humide et sous application prolongée d'un champ électrique positif, le processus dominant consiste en un mouvement des ions $\mathrm{OH}^{-}$, processus qui n'aggrave pas l'instabilité du polymère.

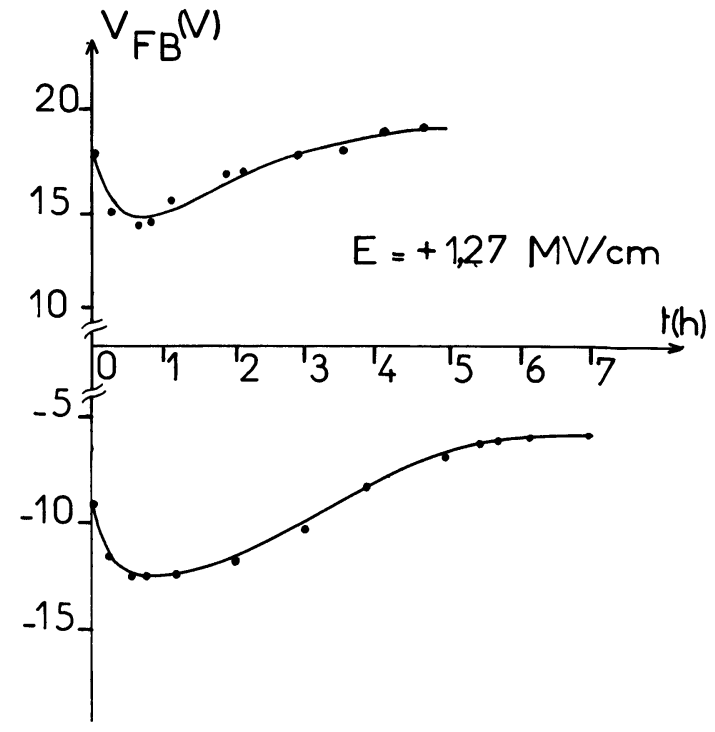

Fig. 13. - Déplacement de la courbe $C(V)$ sous l'action d'un champ de $1,27 \times 10^{6} \mathrm{~V} / \mathrm{cm}$ et d'une humidité relative de $80 \%$.

[Shift of the $C(V)$ curve under the effects of a field of $1.27 \times$ $10^{6} \mathrm{~V} / \mathrm{cm}$ and $80 \%$ of relative humidity.]

En effet, on assiste à une compensation des ions $\mathrm{OH}^{-}$ due à l'exposition de la structure M-P-S à une humidité importante et des ions positifs présents dans la couche de polymère et inhérents à sa fabrication.

\section{Conclusion.}

Les interfaces GaAs isolant ont fait l'objet de nombreuses études. Un fait remarquable ressort de ces travaux : la surface de GaAs reste relativement insensible à la nature du diélectrique déposé.

A la lumière de ces résultats, nous avons abordé le problème de la passivation de GaAs en élaborant une couche de diélectrique obtenue par dépôt dans un plasma froid à partir d'une vapeur de polysiloxane.

- Le taux de croissance des couches déposées (1 $000 \AA / \mathrm{min}$.) est du même ordre que ceux observés lors de la formation de films de type nitrure ou oxyde.

- Les propriétés électriques du film diélectrique (courant de fuite $<10^{-10} \mathrm{~A}$, champ de rupture diélectrique de l'ordre de $5 \times 10^{6} \mathrm{~V} / \mathrm{cm}, \varepsilon_{\mathrm{R}}=2.5$ ) en font un excellent isolant.

Le fait que l'on n'observe pas une augmentation de courant avant le claquage en fait de ce point de vue un meilleur isolant que les films de nitrure ou d'oxyde.

- L'adhérence du film déposé est excellente et résiste bien à des chocs thermiques de forte amplitude.

- Les propriétés électriques de l'interface polysiloxane-GaAs présente des performances comparables aux résultats généralement observés sur des 
systèmes diélectrique-GaAs. Même sous $80 \%$ d'humidité relative les dérives de $C(V)$ observées ne montrent pas de variation sensible de la quantité de charges déplacées.

\section{Remerciements.}

Les auteurs remercient le Centre National d'Etudes et Télécommunication du support financier accordé pour cette étude.

\section{Bibliographie}

[1] Spicer, W. E., Landau, I., Pianetta, P., Chye, P. W., GARner, C. M., Thin Solid Films 56 (1979) 1.

[2] Brosset, D., Bui, A., Segui, Y., Appl. Phys. Lett. 33 (1978) 187.

[3] Holahan, J., Bell, A. T., Techniques and Applications of plasma chemistry (J. Wiley) 1974.

[4] Topics in current chemistry, Vol. 94, Plasma Chemistry III (Springer-Verlag) 1980.

[5] Segui, Y., Ai BuI, J. Appl. Polym. Sci. 20 (1976) 1611.

[6] Voir par exemple : Daniel, V., Dielectric Relaxations (Academic Press) 1967.

[7] Wintle, H. J., J. Non. Cryst. Solids 15 (1974) 471.

[8] Sawada, S., Hasegawa, H., Thin Solid Films 56 (1979) 183.
[9] Yokoyama, S., Yukitomo, K., Hirose, M., Osaka, Y., Thin Solid Films 56 (1979) 81.

[10] Terman, L. M., Solid State Electron 5 (1962) 285.

[11] Hasegawa, H., Sawada, T., IEEE Trans. E.D. 27 (1980) 1055.

[12] Special issue on Semiconducting III-V compound M.I.S. structures. Thin Solid Films 56 (1979).

[13] Pu, N. F., Robinson, G. Y., J. Appl. Phys. 53 (1982) 416.

[14] Gourrier, S., Mircea, A., Bacal, M., Thin Solid Films 65 (1980) 315.

[15] Sawada, T., Hagesawa, H., Thin Solid Films 56 (1979) 315.

[16] Bayraktaroglu, B., Johnson, R. L., J. Appl. Phys. 52(5) (1981). 